\title{
Asteroidal $I, J, K$ magnitudes recovered in the DENIS Survey: First release $^{\star}$
}

\author{
A. Baudrand ${ }^{1,2}$, A. Bec-Borsenberger ${ }^{2}$, J. Borsenberger $^{3}$, and M. A. Barucci ${ }^{1}$ \\ 1 DESPA, Observatoire de Paris, France \\ 2 IMCCE, UMR CNRS 8028, Observatoire de Paris, France \\ 3 Institut d'Astrophysique de Paris, France
}

Received 12 September 2000 / Accepted 4 April 2001

\begin{abstract}
The DENIS programme (Deep European Near-Infrared southern sky Survey) is carrying out a groundbased survey of the southern sky with the aim of providing an extensive $I, J, K$ photometric catalog of point and extended sources. The limiting magnitudes of the three bands $I, J, K$ centered at $0.8,1.25$ and $2.15 \mu \mathrm{m}$ are respectively 18.5, 16.5 and 13.5. With respect to the exposure time of the observations, asteroids are included in the point source catalog as any other regular point-like object. Up to now, we have identified 1233 asteroids, numbered between 1 and 8000, on the basis of their predicted positions following a recognition procedure described here. Their $I, J, K$ magnitudes are being compiled in electronic tables available at the CDS via anonymous ftp 130.79 .128 .5 .
\end{abstract}

Key words. asteroids - infrared: solar system - survey

\section{Introduction}

Up to recently, few near-infrared data were available for asteroids even though this spectral range may contribute significantly to the mineralogical studies of their surface. Asteroids have been grouped on the basis of colors, primarily at visual wavelengths, into different classes (Tholen 1984; Barucci 1987; Tedesco et al. 1989; Bus 1999). These classes roughly correspond to certain kinds of mineralogies. Previous near-infrared studies (Veeder et al. 1982, 1983a, 1983b, 1995) have indicated that some of these distinctions carry from visual wavelengths into the nearinfrared, so some of these classes might be distinguishable on the basis of their near-infrared colors alone. Presently, the 2MASS Asteroid and Comet Survey (Sykes et al. 2000), has made available a large and uniform body of asteroid $J H K$-photometry.

The DENIS programme, since December 1995, has been carrying out a near-infrared survey which is primarily dedicated to stars and galaxies. However, many asteroids are being intercepted during the observations and included in the photometric $I J K$-catalog of point sources, which is one of the DENIS final products. As we expected that

Send offprint requests to: A. Baudrand, e-mail: baudrand@bdl.fr

* Tables 3, 4 and 5 are only available in electronic form at the CDS via anonymous ftp to

cdsarc.u-strasbg.fr(130.79.128.5) or via

http://cdsweb.u-strasbg.fr/cgi-bin/qcat?J/A+A/375/275 the complete survey would serendipitously detect many thousands of asteroids, DENIS appeared to us as a large potential reservoir to extract an unprecedented uniform body of $I J K$-photometry of asteroids. In the DENIS data stream, the recognition of asteroid data was not trivial and we had to build an interface, between the DENIS observations and the numbered asteroids, which computes their ephemerides and compares them with the paths and epochs of the DENIS sky scans. Another module was created to process in the catalog database the search for DENIS sources positionally correlated with the predicted positions of the selected candidates and to release their corresponding apparent magnitudes in the three bands. DENIS observations are presented in Sect. 2. Then, we explain the procedure that we used for recovering the asteroids (Sect. 3). The results are discussed in Sect. 4 and are provided in the electronic tables.

\section{DENIS observations, reduction and catalog}

\subsection{Survey observations}

DENIS is a European joint programme which is carrying out a highly uniform digital imaging survey of the southern sky (between, $+2^{\circ}$ and $-88^{\circ}$ of declination), simultaneously in three near-infrared bands, $I(0.82 \mu \mathrm{m})$, $J(1.25 \mu \mathrm{m})$, and $K \mathrm{~s}(2.15 \mu \mathrm{m})$. The limiting magnitudes in each band are respectively, 18.5, 16.5, 13.5 to a sensitivity at about 2 sigma. The observations are performed 


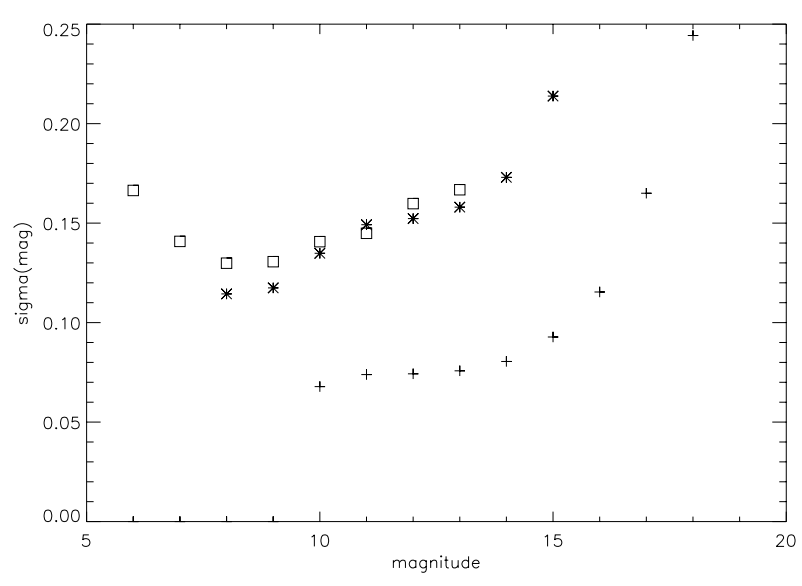

Fig. 1. Magnitude accuracies in each band $I(+), J\left(^{*}\right), K$ (square) are shown as functions of the magnitude (G. Simon, private communication).

with the $1 \mathrm{~m}$-ESO telescope at La Silla (Chile) dedicated to the programme and equiped with a 3-channel camera. The survey observing mode consists of scanning the sky in strips of $30^{\circ}$ in declination and $12^{\prime}$ in right ascension. A strip is made of 180 images $\left(12^{\prime} \times 12^{\prime}\right)$ whose exposure time is 10 seconds and two consecutive frames are $2^{\prime}$ overlapped.

The observations started at the end of 1995 and should be completed by mid-2001. Up to now, $90 \%$ of the southern sky has been scanned.

\subsection{Reduction pipeline}

A highly automated software pipeline processes the raw data and produces the final survey products which will ultimately include a Point Source Catalog, an Extended Source catalog and an Image Atlas of digital $12^{\prime} \times 12^{\prime}$ images in the three survey colors. The Paris Data Analysis Center (PDAC) is in charge of the processing of the raw data (flat-fielding, sky substraction, photometric calibration) and of the archieving of the processed data in accessible databases. The extraction of point-like objects and the astrometry were first achieved at the Leiden Data Analysis Center (LDAC) and are now performed officially at the PDAC, following the latest improvements.

The position of a general extracted point source is provided with an accuracy better than $1^{\prime \prime}$ with respect to the USNO2 catalog and its $I, J$ and $K$ magnitudes to better than 0.2 mag (see Fig. 1).

For more detailed information, the reader is referred to the following website pages of DENIS:

- http://www-denis.iap.fr

- http://cdsweb.u-strasbg.fr/denis.html

- http://pcnt1.iap.fr/Denis/pdacdoc-fr.html.

\subsection{The point source catalog}

One of the final DENIS products is the point source catalog accessible as a multi-entry database. For each extracted object, the following items are compiled:

- its DENIS identificator;

- the strip number where it was extracted;

- its right ascension and declination (geocentric, J2000);

- its $I, J, K$ magnitudes;

- the $I, J, K$ photometric rms errors;

- its visual USNO2 counterpart, if any.

These photometric rms errors are estimated as a function of magnitude, and derived from sources seen more than once in a strip (approximately $10 \%$ of the sources, located in the overlaps at the north and south of the images).

A preliminary catalog of the objects of 478 strips from 1996 was released by the LDAC in december 1998, corresponding to about $10 \%$ of the sky. The PDAC is now providing daily the official database catalog at the same speed as the scanning of the sky. Up to now, it has already made accessible another $47 \%$ of the sky.

\section{Asteroid recovering procedure}

The following procedure determines which known asteroids fall within each survey scan on a given night and then searches for DENIS detections which are positionally correlated with the predicted positions of those objects.

\subsection{Tools and performances}

Asteroids can be retrieved on purely positional criteria thanks to robust ephemerides and astrometry. Indeed, the astrometric precision of DENIS point source positions is typically better than $1^{\prime \prime}$ and the ephemerides of the asteroids are computed with an accuracy better than $1^{\prime \prime}$. However, with regard to the quality of the determination of the initial conditions, typical uncertainties are in the range 1-5 arcsec. The ephemerides are computed thanks to numerical integration and Chebychev Polynomial approximations of a 10-body problem (Bec-Borsenberger 1992). The orbits of the planets were included for completeness and were taken from the JPL DE403 (Standish 1995). As initial conditions, we use the osculator elements published in the Asteroid Orbital Elements Database for all numbered asteroids (Bowell 1999). This database also provides their CEU (Current Ephemeris Uncertainty) and their greatest PEU (Peak Ephemeris Uncertainty) within 10 years. We reported them in the table of results (Sect. 4.1, Table 2).

\subsection{Selection among observed strips}

In a first step, we expand the $\left(12^{\prime} \times 30^{\circ}\right)$-scan box by $3^{\prime}$ at each corner on both axes and we calculate the Julian date of the scan midpoint time. The position of thousands of numbered asteroids are calculated for all these dates so that we can select which of them fall within the expanded scan boundaries at the specified epoch. The size of the box expansion allows the inclusion of minor planets having fairly large angular motion over a typical scan duration. 
As a second step, for each of these pre-selected asteroids, we compute its ephemerides, with a 5 min step, during the scan of the strips which have roughly intercepted it. Then, the final selection is obtained by comparing these positions with the non-expanded strip box.

This two-step procedure was necessary to reduce the computing time of integration without replacing the 10 -body problem by a 2 -body problem.

\subsection{Detection in the catalog and validation of the associations}

For the whole set of the selected candidates, an automated request to the point source catalog processes the search for sources within a coarse disk window of $20^{\prime \prime}$ radius around the predicted positions. The radius was chosen to be large enough to view the field surrounding the asteroid.

In addition to the items given by the catalog (cf. 2.3), the distances between the point sources selected $\left(\mathrm{RA}_{1}\right.$, $\left.\mathrm{DEC}_{1}\right)$ and the asteroid positions predicted $\left(\mathrm{RA}_{2}, \mathrm{DEC}_{2}\right)$ are also provided by:

$d=\sqrt{\left(\mathrm{DEC}_{1}-\mathrm{DEC}_{2}\right)^{2}+\left(\mathrm{RA}_{1}-\mathrm{RA}_{2}\right)^{2} \cdot \cos \mathrm{DEC}_{2}}$.

For each asteroid, we select the DENIS point sources which follow the conditions below:

- they are found in the predicted strip;

- they have no visual USNO2-counterpart;

- their minimum distance to the asteroid during the strip, $d_{\min }$, is smaller than $5^{\prime \prime}$ with regard to the precisions and uncertainties on positions (cf. 3.1);

- their $V-I$ index ranges in realistic boundaries: $|V-I| \leq 3$.

From this final selection, each asteroid has been associated with one or several DENIS sources. In order to avoid misassociations, we reject the association when the asteroid is associated with three or more sources and we validate it when only a single source is associated. In the case of two sources associated, they are most of the time one single source observed twice in the overlap of two consecutive images and therefore have nearly the same magnitudes. If such a case is verified, we validate the association. If not, we reject it.

\section{The results: $I, J, K$ magnitudes}

\subsection{Description of the data}

The data reported here and summarized in Table 1, result from the previous procedure (Sect. 3) computed for the first 8000 numbered asteroids and with the 2380 strips available in the catalog database in January 2001.

Out of the 2107 interceptions predicted with the selection procedure (cf. 3.2), 1385 were positively associated with a DENIS source of the catalog. Finally, considering the multiple detections of the same object (152 doubles), 1233 different asteroids were recovered and are compiled in three electronic tables with all the items
Table 1. Number of asteroids detected one or two times and number of associations validated.

\begin{tabular}{llll}
\hline & one time & two times & associations \\
\hline$I$ only & 257 & 29 & 315 \\
$I$ and $\mathrm{J}$ only & 542 & 75 & 692 \\
$I, J$, and $K$ & 282 & 48 & 378 \\
\hline Total & 1233 & 152 & 1385 \\
\hline
\end{tabular}
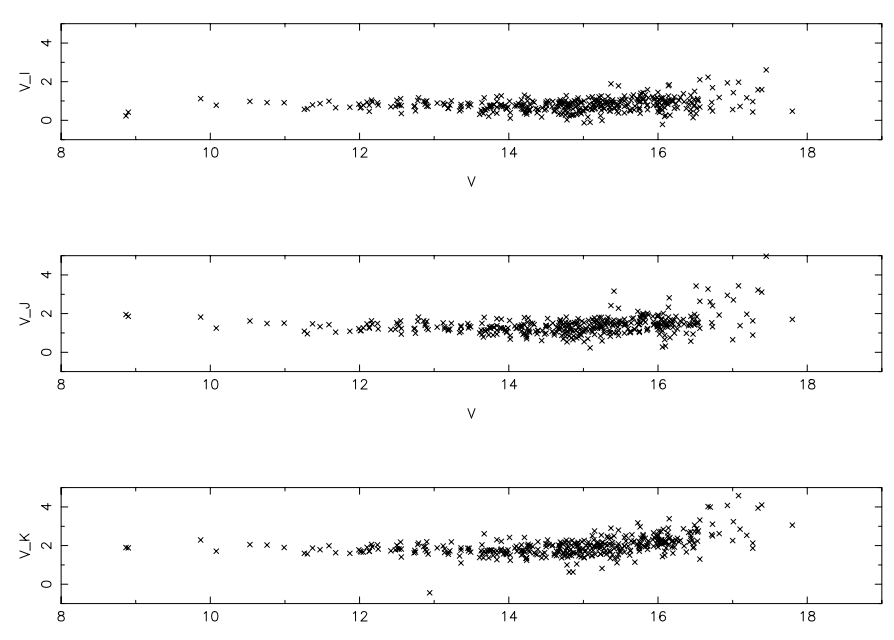

Fig. 2. The differences between predicted $V$ magnitudes and observed $I, J$ and $K$ magnitudes for DENIS sources associated with known asteroids are shown as functions of the predicted $V$ magnitudes.

described in Table 2 :

- the detections recovered in the three colors are reported in the electronic Table 3;

- the detections with missing colors are reported in the electronic Tables 4 (only $I$ and $J$ ) and 5 (only $I$ ).

Tables 3,4 and 5 are available at the CDS via anonymous ftp 130.79 .128 .5 .

\subsection{Reliability of the data}

Although the risk of misassociation is minimized by the validation step (cf. 3.3), asteroids exhibiting unusual nearinfrared colors have to be examined very cautiously, as one cannot interpret the colors of individual asteroids without considering the possibility of contamination by a background source.

As shown in Fig. 2, asteroids color indexes are likely to range between the following boundaries:

$0<V_{\text {pred }}-I<+1.5$

$+0.5<V_{\text {pred }}-J<+2$

$+1<V_{\text {pred }}-K<+3$.

Out of these ranges, the DENIS counter-part may result from the superposition of the asteroid and a background star. However, a large acceptance window is needed because of unknown color effects and uncertainties in absolute magnitudes. 
Table 2. Column description of the electronical DENIS asteroid Tables 3, 4 and 5.

\begin{tabular}{lll}
\hline Column & Name & Description \\
\hline 1 & $N_{\text {ast }}$ & Asteroid number \\
2 & $N_{\text {strip }}$ & DENIS strip number \\
3 & Datejj & Julian date of the observation \\
4 & Dgeo & Geocentric distance $(\mathrm{au})$ \\
5 & $V_{\text {pred }}$ & Predicted $V$ magnitude \\
6 & PHI & Phase angle (deg) \\
7 & Dhel & Heliocentric distance (au) \\
8 & $N_{\text {ima }}$ & DENIS image number \\
9 & RA & Right Ascension (J2000) of DENIS source (deg) \\
10 & DEC & Declination (J2000) of DENIS source (deg) \\
11 & $I$ & $I$-band magnitude \\
12 & $J$ & $J$-band magnitude \\
13 & $K$ & $K$-band magnitude \\
14 & Err $_{I}$ & $I$-band photometric rms error (cf. 2.3) \\
15 & Err $_{J}$ & $J$-band photometric rms error (cf. 2.3) \\
16 & Err $_{K}$ & $K$-band photometric rms error (cf. 2.3) \\
17 & Dist & Angular distance (arcsec) between the predicted position \\
& & of the asteroid and its DENIS counter-part (cf. 3.3) \\
18 & CEU & Current Ephemeris Uncertainty (arcsec) (cf. 3.1) \\
19 & PEU & greatest Peak Ephemeris Uncertainty within 10 years (arcsec) (cf. 3.1) \\
\hline
\end{tabular}
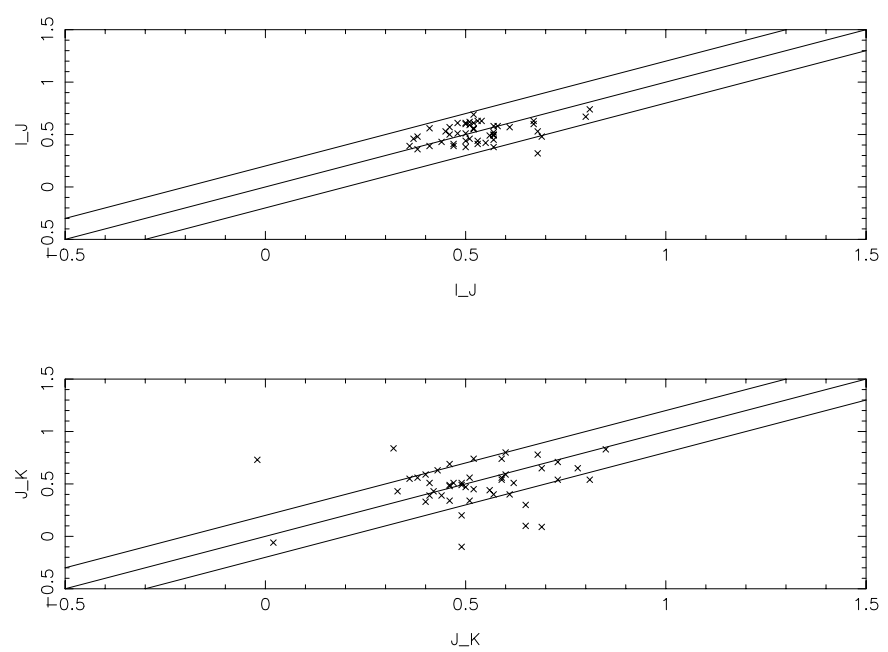

Fig. 3. Comparisons of the $J-K$ values and the $I-J$ values for the 48 asteroids observed twice by DENIS in the three bands on two different dates.

One cannot sensibly compare observed asteroid magnitudes obtained at different epochs, because their brightnesses are highly variable both with heliocentric and geocentric distances and as a consequence of lightcurve variations. However, to the extent that their surfaces are compositionally homogeneous and that the phase effect is not taken into account, colors should not change. Therefore, internal comparisons have been made possible thanks to the detections at two different dates of 123 asteroids in $I J K$ or only $I J$. They are presented in

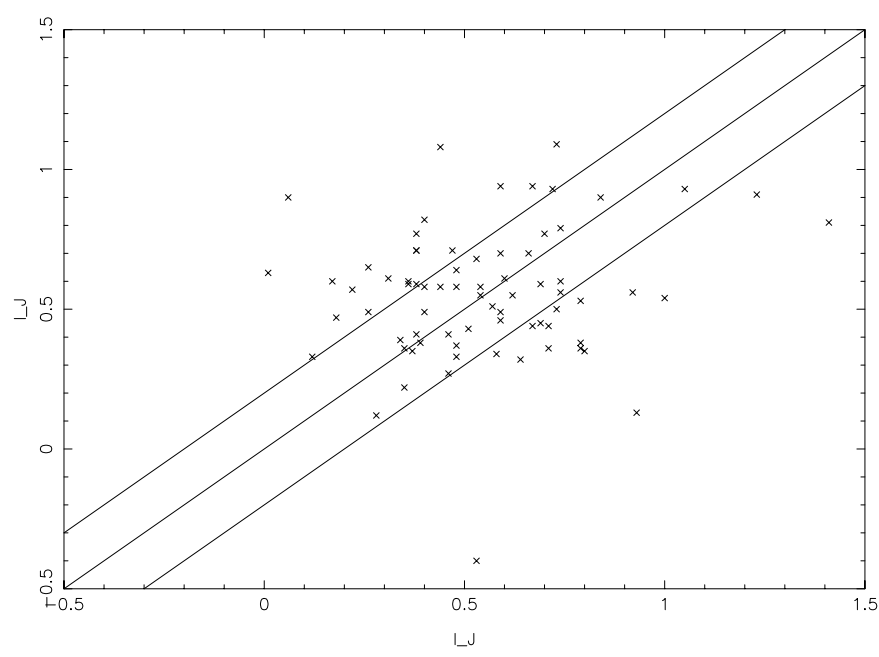

Fig. 4. Comparisons of the $I-J$ values for the 75 asteroids observed two times by DENIS in the $I$ and $J$ bands at two different dates.

Figs. 3 and 4 which show a good internal agreement, as most of the values fall inside the uncertainty boundaries. However, we note that the comparisons are more satisfactory for asteroids observed in the three bands than for those recovered only in $I$ and $J$, as they were too faint in $K$. It is due to the degradation of the photometric accuracies as magnitudes increase (see Fig. 1). 


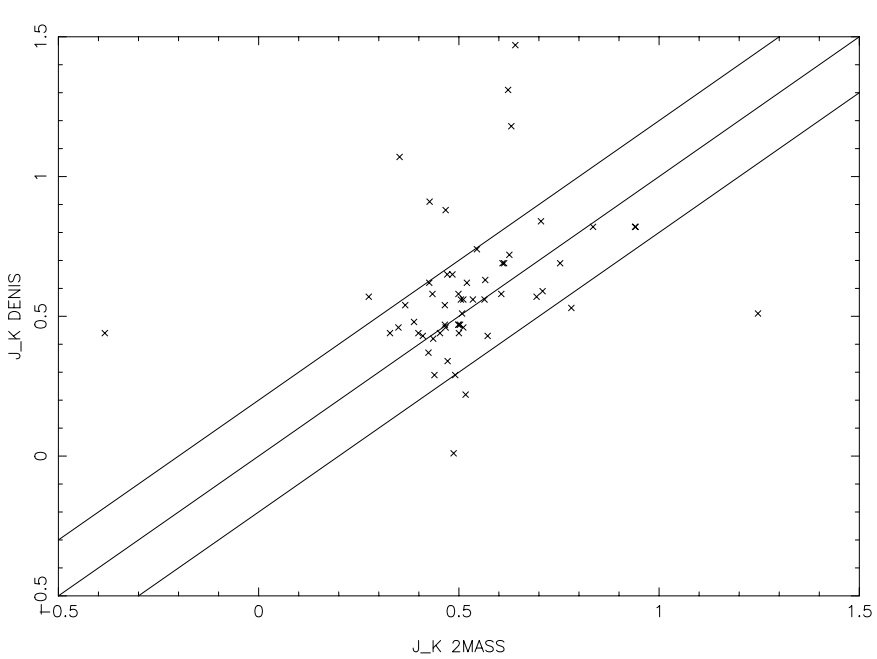

Fig. 5. $J-K$ comparisons of 58 objects commonly observed by DENIS and 2MASS surveys.

\subsection{Comparisons with other datasets}

Any dataset obtained by one survey has been obtained through a specific set of observation and reduction strategies. Still, colors of common objects can be compared.

Like DENIS, the 2MASS survey (Cutri et al. 1999) is currently scanning the whole sky, in the aim of an extensive $J H K$ photometric catalog of stars and galaxies. It has also released a large asteroid dataset (Sykes et al. 2000). 58 asteroids were commonly observed by DENIS and 2MASS and their $J-K$ colors have been compared (Fig. 5). The differences found are inside the uncertainty boundaries and so, quite satisfactory.

Two other $J H K$ datasets were used for comparison with DENIS: one sample of 151 objects (Hahn \& Lagerkvist 1988) and an other of 56 objects (Veeder et al. 1995). The comparison for the 50 objects in common are reported in Fig. 6 and also show a good agreement.

\section{Conclusion}

We have made 1385 associations of asteroids with DENIS sources, out of which 1233 different asteroids have been identified among the 8000 first asteroids. With regard to the size of the samples used up to now for the mineralogical classifications (Zellner 1985; Bell 1988; Tedesco et al. 1992; Xu 1995) this number is quite encouraging. Moreover, it was obtained with only $47 \%$ of the survey.

The asteroid retrieving is being continued with more observations and more small bodies which are now numbered up to nearly 12000 . We have already predicted more than 1088 other detections of asteroids - numbered between 1 and 8000 - among 1593 other strips, which corresponds to another $30 \%$ of the survey. As soon as these observations are included in the catalog database, the automated requests will be relaunched to extract additionnal $I, J, K$ magnitudes.

DENIS overlaps and extends existing observations of asteroids from the visible into the near-infrared, where

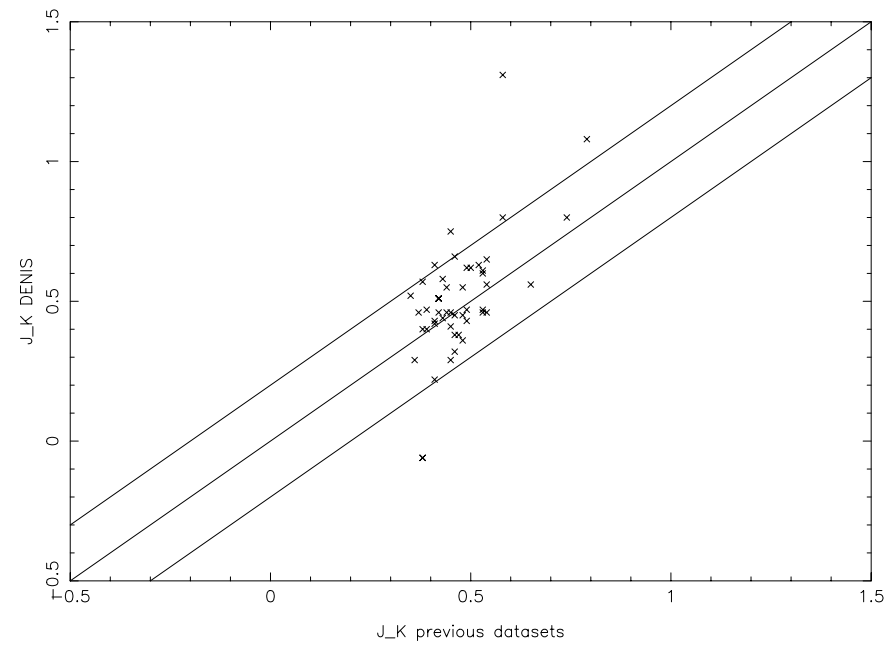

Fig. 6. $J-K$ comparisons of 50 objects commonly found in the DENIS catalog and previous $J H K$ asteroid datasets (Hahn \& Lagerkvist 1988; Veeder et al. 1995).

there exist important features arising from common minerologies (Gaffey et al. 1993). Thus, this new large set of data will allow for potentially significant refinements to asteroid classifications (Bus 1999).

Acknowledgements. The authors thank the referee, Mark Sykes, for his corrections and his very useful comments in comparing the data. We also thank Guy Simon from the DENIS team for his help as well in comparison questions. Finally, we thank the DENIS staff and all the DENIS observers who collected the data. The DENIS programme is partly funded by the European Commission through SCIENCE and Human Capital and Mobility grants. It is also supported in France by INSU, the Education Ministry and CNRS, in Germany by the Land of Baden-Wurtenberg, in Spain by DGICYT, in Italy by CNR, in Austria by the Fonds zur Förderung der Wissenschaft und Forschung, in Brazil by FAPESP.

\section{References}

Baudrand, A., Bec-Borsenberger, A., Borsenberger, J., \& Barucci, M. A. 1999, Asteroidal recovering in the DENIS survey, in Evolution and Source Regions of Asteroids and Comets, Proc. IAU Coll., 173, 169

Barucci, M. A., Capria, M. T., Coradini, A., \& Fulchignoni, M. 1987, Icarus, 72, 304.

Bec-Borsenberger, A. 1992, A\&A, 258, 94

Bell, J. F., Owensby, P. D., Hawke, B. R., \& Gaffey, M. J. 1988, in Proc., 19th Lunar Planet Sci. Conf., 57

Bowell, E. 1999, The Asteroid Orbital Elements Database, ftp://ftp. lowell.edu/pub/elgb/astorb.html

Bus, S. J. 1999, Compositional Structure in the Asteroid Belt: Results of a spectroscopic survey, Ph.D. Thesis, Massachusetts Institute of Technology

Cutri, R. M., Skrutskie, M. F., Van Dyck, S., et al. 1999, Explanatory Supplement to the 2MASS Spring 1999 Incremental Data Release, http://www.ipac.caltech.edu/2mass/releases/spr99/ doc/explsup.html 
Gaffey, M. J., Bell, J. F., Brown, R. H., et al. 1993, Icarus, 106, 573

Hahn, G., \& Lagerkvist, C.-I. 1988, Icarus, 74, 454

Standish, E. M., Williams, J. G., \& Folkner, W. M. 1995, JPL Planetary and Lunar Ephemerides, DE403/LE403, JPL IOM, 314, 10

Sykes, M. V., Cutri, R. M., Fowler, J. W., et al. 2000, Icarus, 146,161

Tedesco, E. F., Williams, J. G., Matson, D. L., et al. 1989, AJ, 97,580

Tedesco, E. F., Veeder, G. J., Fowler, J. W., \& Chilleni, J. R. 1992, The IRAS Minor planet Survey, Phillips Laboratory PL-TR-92-2049
Tholen, D. J. 1984, Ph.D. Asteroid taxonomy from cluster analysis of photometry, Doctoral Thesis, Univ. of Arizona Veeder, G. J., Matson, D. L., \& Kowal, C. 1982, AJ, 87, 834

Veeder, G. J., Matson, D. L., \& Tedesco, E. F. 1983a, Icarus, 55,177

Veeder, G. J., Matson, D. L., Hoover, G., \& Kowal, C. 1983b, AJ, 88, 1060

Veeder, G. J., Matson, D. L., Owensby, P. D., et al. 1995, Icarus, 114, 186

Xu, S., Binzel, R. P., Burbine, T. H., \& Bus, S. J. 1995, Icarus, 115,1

Zellner, B., Tholen, D. J., \& Tedesco, E. F. 1985, Icarus, 61, 355 